\title{
REMOÇÃO DE TERCEIRO MOLAR EM SEIO MAXILAR COM USO DE TÉCNICA MINIMAMENTE INVASIVA: RELATO DE CASO
}

\section{Lemos Felicio Agostinho Cicero Newton ${ }^{1 *}$, Dias dos Santos Thais ${ }^{1}$, Viana Araújo Leonardo ${ }^{2}$}

DOI: 10.48018/rmv.v31.i2.13

Este artículo está bajo una licencia de Creative Commons de tipo Reconocimiento - No comercial - Sin obras derivadas 4.0 International.

\section{Resumo}

O seio maxilar é uma estrutura anatômica pneumatizada e localizada na região anterior da maxila bilateralmente. A ocorrência do deslocamento de corpos estranhos no seio maxilar não é uma condição comum, e quando presente está associada a acidentes automobilísticos, acidentes por arma de fogo, distúrbios psiquiátricos e iatrogenias em procedimentos cirúrgicos. A remoção do corpo estranho é realizada a fim de prevenir infecções, podendo a permanência do corpo estranho oferecer danos imediatos ou crônicos. $O$ acesso ao seio maxilar é realizado mais comumente pela técnica de acesso Caldwell-Luc. Desta forma, o objetivo do estudo é relatar um caso clínico de remoção cirúrgica de um terceiro molar superior do interior do seio maxilar pela técnica de acesso Caldwell-Luc, com auxílio de um guia cirúrgico a fim de conduzir uma cirurgia mais segura e menos invasiva.

Palavras Chave: Seio Maxilar; Terceiro Molar; Cirurgia Bucal; Exodontia.

orcid.org/0000-0002-56583749

Dias dos Santos Thais

orcid.org/00000002-7226-7519

Viana Araújo Leonardo

orcid.org/0000-0002-5756-5446

*Corresponding author: Lemos Felicio Agostinho Cicero Newton

E-mail: ciceronewton@yahoo.com.br

Article history

Received: 14 - Jul - 2020

Accepted: 17 - Sep -2020

Publish: 01 - Oct - 2020

CARE 2017 Check List statement: The authors have real the CARE 2017 Check List and the manuscript was prepared and revised according to the CARE 2017 Checklist.

Conflict of interest: All authors declared that there are no conflicts of interest.

Financial disclosure: The authors have no financial relationships relevant to this article to disclose

Authors' contribution: All the authors contributed in the search, selection of articles and writing. All the authors reviewed and approved the final manuscript.

Forma de citar este artículo: Lemos Felicio CN, Dias dos Santos T, Viana Araújo L. REMOÇÃO DE TERCEIRO MOLAR EM SEIO MAXILAR COM USO DE TÉCNICA MINIMAMENTE INVASIVA: RELATO DE CASO. Rev Med Vozandes. 2020; 31 (2): $96-100$

The maxillary sinus is a pneumatized anatomical structure located bilaterally in the anterior region of the maxilla. The occurrence of displacement of foreign bodies in the maxillary sinus is not a common condition and when present, it is associated with car accidents, fire arm accidents, psychiatric disorders and iatrogenies in surgical procedures. The removal of the foreign body is carried out in order to prevent infections, and the permanence of the foreign body may offer immediate or chronic damage. Access to the maxillary sinus is most commonly performed using the Caldwell-Luc access technique. Thus, the objective of the study is to report a clinical case of surgical removal of a maxillary third molar from the interior of the maxillary sinus using the caldwell-luc access technique, with the aid of a surgical guide in order to conduct a safer and less invasive surgery. Keywords: Maxillary Sinus; Third Molar; Oral surgery; Exodontia. 


\section{Introdução}

O seio maxilar é uma estrutura anatômica pneumatizada e localizada na região anterior da maxila bilateralmente. Essa estrutura é também conhecida como uma cavidade paranasal por estar localizada lateralmente à fossa nasal, seu assoalho frequentemente situa-se 0,5 a 1,5 cm abaixo da cavidade nasal. O mesmo é responsável por filtrar o ar inspirado, aquecê-lo, aliviar o peso do crânio, aliviar impacto em possíveis traumas e dar ressonância à voz. É considerado o maior seio paranasal pois, frequentemente, possui grande volume quando comparado aos demais seios, além de por vezes exibir fragilidade capilar e íntima relação com as raízes dos molares e pré-molares superiores e, por este motivo, oferece risco a complicações cirúrgicas, tais como, a comunicação bucossinusal e o deslocamento de raízes, dentes, implantes e corpos estranhos para o seu interior(1).

Anatomicamente possui formato piramidal quadrangular, no qual seu ápice encontra-se voltado ao processo zigomático da maxila, tendo volume médio, em um paciente adulto, de $14 \mathrm{~cm}^{3}$ de acordo com Ariji e colaboradores (1994). Em até $26,5 \%$ dos pacientes pode possuir septos e cristas em seu interior, encontrados com maior frequência em sua porção média(2).

Histologicamente é revestido por um epitélio respiratório, ou seja, epitélio pseudo-estratificado colunar, ciliado e mucossecretor, considerado, por vezes, um revestimento mucoperiosteal ${ }^{(2,3)}$.

Para Cerqueira(3) o deslocamento de corpos estranhos para o interior do seio é pouco comum e pouco relatado, visto que um terço dos casos não são diagnosticados imediatamente. Quando presente, as principais causas são traumas em face e iatrogenias em procedimentos cirúrgicos odontológicos.

A região posterior da maxila é a mais acometida durante procedimentos cirúrgicos, devido à proximidade das raízes de molares superiores, por vezes já pneumatizado. Tal acometimento se intensifica também devido à qualidade óssea da maxila, que se apresenta predominantemente medular e com pouca espessura de osso cortical, determinando um aspecto poroso, principalmente em pacientes jovens ${ }^{(4)}$.

No deslocamento de raízes ou elementos contaminados, seja por lesões periapicais ou doença periodontal, o risco de infecção sinusal é maior que quando comparado aos deslocamentos de elementos hígidos, podendo gerar reabsorção óssea circunjacente e reação inflamatória no seio maxilar (sinusite), produção excessiva de muco, dor em região periorbitária, halitose, tumefação, coriza e presença de fístula bucosinusal. Esses sintomas geralmente se tornam crônicos, apresentando também episódios agudos recorrentes ${ }^{(3,4)}$.

Alguns exames de imagem são opções viáveis para localização de corpo estranho e para a realização de um planejamento cirúrgico adequado. A ortopantomografia, a incidência de Waters e a perfil de face podem ser empregadas, no entanto, a tomografia computadorizada de face oferece uma imagem tridimensional, possibilitando avaliação e conduta mais apropriada ${ }^{(1,3)}$.

O acesso ao seio maxilar é realizado mais comumente pela técnica de Caldwell-Luc, que foi desenvolvido em 1890 por George Caldwell nos Estados Unidos e Henri Luc na França. O acesso é realizado através da osteotomia da parede anterior, permitindo assim a inspeção do interior do antro e remoção de corpos estranhos. Complicações inerentes a utilização desta técnica são poucos comuns, porém quando presentes podem incluir: assimetria facial, lesão nervosa, desvitalização dentária e fístulas oroantrais $(1,3)$.

A era digital na odontologia tem sido uma realidade para diversas especialidades, tal como a ortodontia, prótese e cirurgias ortognáticas. Recursos digitais e fotografias em 3D tem dado maiores opções de tratamento e oferecido planejamentos de melhor qualidade. A utilização destes recursos pode oferecer maior previsibilidade e conforto ao paciente ${ }^{(5)}$.

Desta forma, o objetivo do estudo é relatar um caso clínico de remoção cirúrgica de um terceiro molar superior, no interior do seio maxilar, pela técnica de acesso caldwell-luc, com auxílio de um guia cirúrgico, com objetivo de orientar o acesso ao corpo estranho de forma rápida e minimamente invasiva, proporcionando uma cirurgia mais segura. $O$ artigo segue as diretrizes CARE para o relato de $\operatorname{casos}^{(6)}$.

\section{Relato de caso}

Paciente AGN, gênero masculino, 17 anos de idade, procedente de São Luís do Maranhão, buscou atendimento com a equipe de Cirurgia e Traumatologia Bucomaxilofacial do Hospita Clementino Moura, após ter observado, junto ao cirurgião-dentista, um "corpo estranho" na região anatômica correspondente ao seio maxilar direito, através de achados imaginológicos de rotina solicitado para início de tratamento ortodôntico. O trabalho foi realizado após aprovação do Comitê de Ética e Pesquisa da sob o CAAE número 26029819.1.0000.5084.

O mesmo não relatava queixas inerentes a condição em que foi observada. Na anamnese o paciente relatou ter sido submetido à tentativa de extração do terceiro molar superior direito (elemento 18) há mais ou menos um ano sem sucesso, neste período não houve nenhum sintoma relatado pelo paciente. O histórico familiar do paciente em questão não mostrou nenhum dado associado a comorbidades, alergias ou doenças tumorais.

Ao exame físico extra oral não foi observada nenhuma alteração digna de nota. Ao exame intra oral, o paciente apresentava-se parcialmente dentado inferior e superior, lábios corados, abertura bucal satisfatória, mucosa intra oral apresentando coloração normal e sem sinais de inflamação e/ou infecção.

Ao exame tomográfico evidenciou-se uma área hiperdensa na região referente ao seio maxilar esquerdo com tamanho aproximado de $12 \mathrm{~mm}$ 
e velamento do mesmo, sugerindo presença de conteúdo líquido a esclarecer em seu interior (Figura 1).

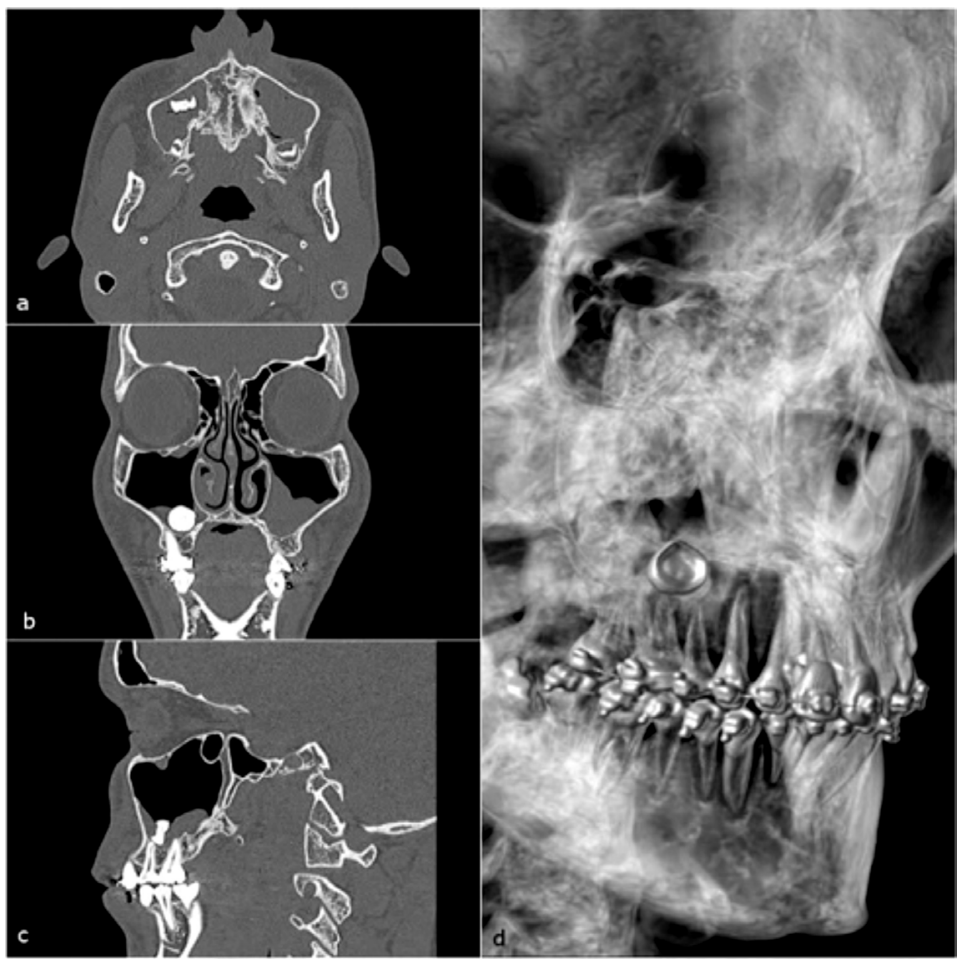

Figura 1. Imagens tomográficas com presença de imagem sugestiva de elemento dentário em seio maxilar direito

Fuente: Elaborado por los autores

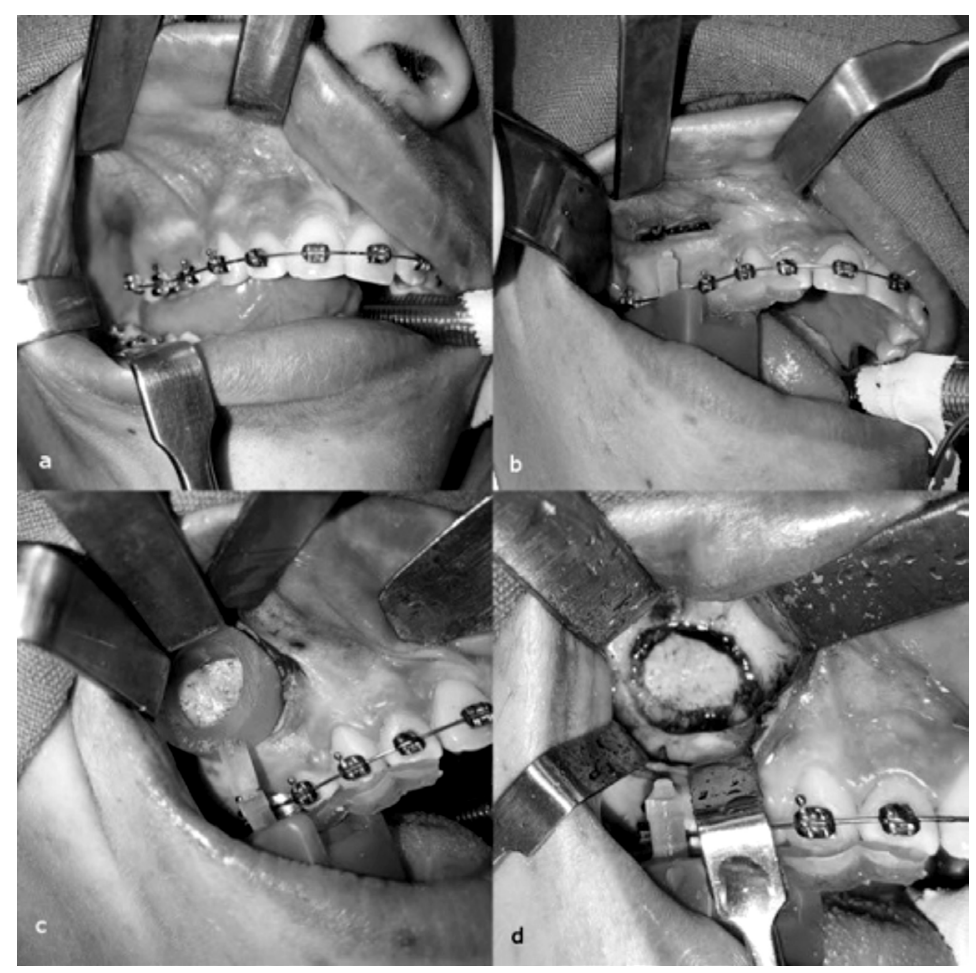

Figura 2. Sequência de incisão, interposição do dispositivo Localizador e osteotomia da parede anterior do seio maxilar

Fuente: Elaborado por los autores
O planejamento cirúrgico preconizou uma intervenção sob anestesia geral que oferecesse menor trauma aos tecidos locais, e que proporcionasse maior segurança e menor tempo cirúrgico. Desta forma foi planejada a confecção de um guia cirúrgico com objetivo de orientar o acesso ao corpo estranho de forma rápida e minimamente invasiva.

Para isso, o paciente foi encaminhado à uma clínica radiológica para escaneamento digital intraoral das arcadas superior e inferior, e posterior confecção de modelos digitais. Com estes modelos em associação com a tomografia computadorizada, utilizou-se o arquivo DICOM (Digital Imaging and Communications in Medicine) para início da confecção do guia.

Inicialmente os arquivos digitais foram manipulados no software Nemo Studio (Nemotec) para unir os modelos digitais ao exame tomográfico com objetivo de criar um primeiro componente do dispositivo que se encaixasse na superfície oclusal dos elementos superiores. Além disso foi realizado o processo de segmentação do corpo estranho e das raízes dos dentes adjacentes, que consiste na individualização e criação de arquivos digitais criados a partir do arquivo DICOM, permitindo assim a visualização e manipulação desses componentes de maneira tridimensional, sendo possível analisar a relação espacial entre o corpo estranho e as estruturas anatômicas da região de interesse.

No segundo momento foi utilizado o software Meshmixer (Autodesk) para criação do segundo componente do dispositivo. A tomografia, o modelo digital da arcada superior e o corpo estranho segmentado foram exportados do software Nemo Studio no formato de arquivo STL (Stereolithography), arquivo universal que pode ser manipulado na maioria dos softwares CAD (Computer Aided Design). No Meshmixer foi criado um cilindro posicionado sobre a anatomia do crânio na região correspondente ao corpo estranho para orientar a marcação e abertura da janela de acesso ao seio maxilar. Foi desenvolvido também um sistema de encaixe entre o guia de encaixe sobre os dentes e o guia cilíndrico, visto que o paciente ainda apresentava aparelho ortodôntico, sendo inviável a criação de um dispositivo único.

Após a criação dos dois componentes do dispositivo, estes foram materializados através da tecnologia CAM (Computer Aided Manufacturing) por meio de uma impressora 3D que realiza a construção do objeto por um processo aditivo, ou seja, resina líquida é polimerizada por um feixe de laser camada a camada até a sua completa materialização.

Com o dispositivo confeccionado foi possível dar início ao procedimento cirúrgico. Após ficar submerso em solução aquosa de clorexidina $0,2 \%$, com o objetivo de realizar a antissepsia, o guia cirúrgico foi posicionado sobre a oclusal dos elementos póstero-superiores. Uma incisão 
de aproximadamente $4 \mathrm{~cm}$ foi realizada na mucosa alveolar na altura dos pré-molares superiores à esquerda, seguido do descolamento mucoperiosteal, acessando desta forma a parede anterior do seio maxilar, tal como preconiza a técnica de acesso Caldwell-Luc. Após a incisão e descolamento, o guia foi reposicionado e através de motor com broca cirúrgica de formato esférico número 04 a osteotomia foi realizada respeitando as proporções do dispositivo para melhor localização e apreensão do dente deslocado (Figura 2).

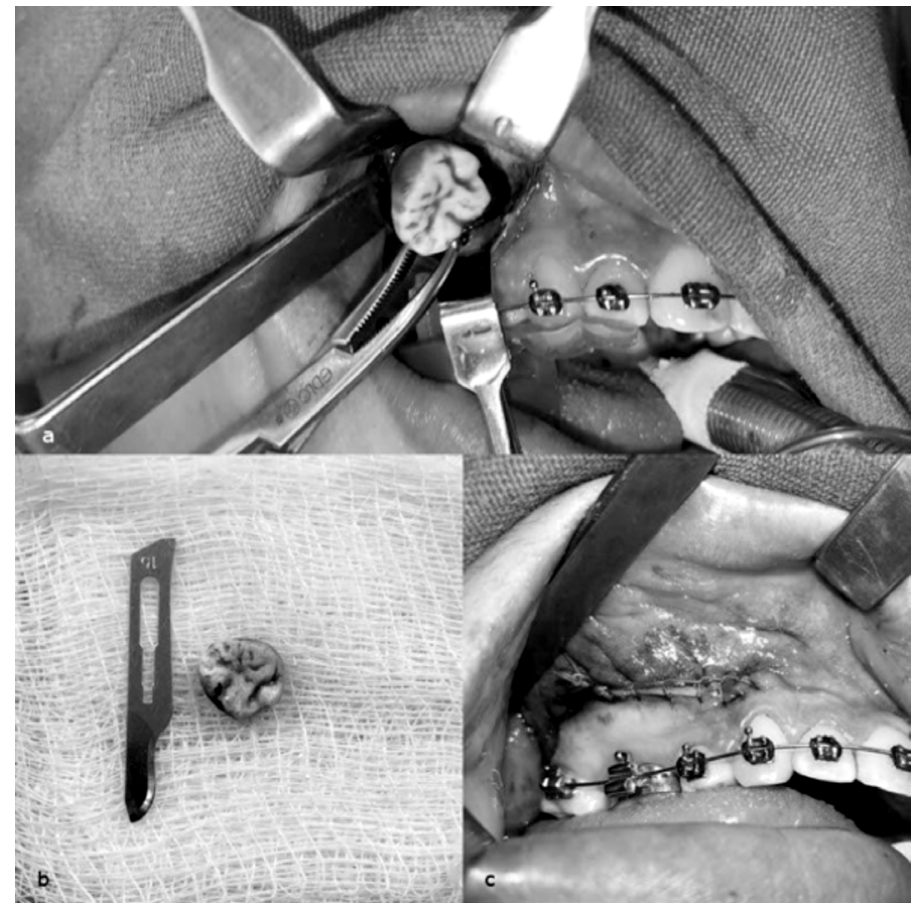

Figura 3. Apreensão da coroa do dente incluso e sutura do. Acesso minimamente invasivo

Fuente: Elaborado por los autores

Finalizada a osteotomia, o guia cirúrgico foi removido e sobre o leito cirúrgico logo foi observado o elemento dentário, seguido da apreensão e exérese do mesmo. A limpeza da área foi realizada com soro fisiológico 0,9\%, remoção do tecido circunjacente e em seguida realizada a síntese com fio absorvível do tipo Vicryl 3-0 (Figura 3).

O paciente encontra-se em acompanhamento pósoperatório por um ano, o mesmo não relata queixas inerentes ao procedimento. As estruturas ósseas e teciduais compatíveis com aspecto de normalidade, oclusão mantida, abertura bucal satisfatória e ausência de tumefação.

\section{Discussão}

A presença de corpo estranho localizado dentro do seio maxilar é pouco comum ${ }^{(3)}$, no entanto, seja por traumas em face ou iatrogenias em procedimentos cirúrgicos, a parede do seio maxilar pode ser frequentemente violada ${ }^{(7)}$. Raízes dentárias, materiais endodônticos, amálgama e implantes são os materiais mais encontrado ${ }^{(8)}$, destacam-se os casos de deslocamentos de terceiros molares superiores ${ }^{(9)}$. A posição dos terceiros molares erupcionados distalmente ao seio maxilar diminui o risco do seu deslocamento, diferentemente das raízes residuais ${ }^{(10)}$.

A menor densidade e maior elasticidade do osso maxilar em pacientes jovens, propicia um risco mais elevado de deslocamento acidental de terceiros molares superiores, tal como o paciente neste caso relatado, importante observar que o estado hígido do elemento dentário, ou seja, livre de cárie ou doença periodontal, e seu deslocamento para o seio maxilar não determina, obrigatoriamente, uma infecção (11). A remoção do corpo estranho é realizada a fim de prevenir infecções futuras, podendo sua permanência oferecer danos inflamatórios agudos ou crônicos ${ }^{(12)}$.

Acerca da curetagem do seio maxilar e da remoção total da sua membrana, há necessidade de remoção apenas se o mesmo, estiver infectado. A remoção imediata do corpo estranho pode evitar possíveis complicações, desta forma podendo ser mantida a integridade da membrana mucoperiosteal ${ }^{(13)}$. Não se observou infecção no caso presente, desta forma se preconizou não realizar a curetagem total do seio maxilar, apenas a remoção do corpo estranho, limpeza cirúrgica da cavidade e remoção do tecido comprometido circunjacente. Cerca de 5-10\% das sinusites maxilares são de causas dentárias (14).

A técnica de acesso Caldwell-Luc ainda é a mais bem sucedida, ainda que existam técnicas assistidas por meio de endoscópios. Este acesso permite visualização satisfatória do seio maxilar e oferece segurança ao paciente por ser de fácil reprodução pelo profissional (12). Dentre as possíveis complicações do acesso encontram-se a epistaxe, injúrias à periórbita, danos à musculatura extrínseca do olho e hemorragia orbitária, porém são pouco comuns ${ }^{(14)}$.

No que diz respeito às técnicas assistidas por endoscópios, apesar de proporcionar melhor visualização, baixa morbidade e aceitação do paciente, exibe alto custo no procedimento, o que dificulta a realização desta técnica no serviço público ${ }^{(13)}$. O uso da luz de fibra ótica provinda de um fotóforo, como artifício auxiliar na localização de corpo estranho no seio maxilar, diminui o tempo cirúrgico, visto que a visualização do interior do seio pode encontrar-se dificultada na presença de conteúdo purulento ou proliferação tecidual no seio maxilar ${ }^{(15,16)}$.

Outra técnica, pouco utilizada atualmente, é a transalveolar, na qual é aproveitada a comunicação do alvéolo com o seio maxilar para a captura do corpo estranho. Vale ressaltar que a técnica só deve ser realizada quando sua abertura for maior que o objeto deslocado e quando o mesmo, estiver visível, porém, a pobre visualização do seio maxilar é a principal desvantagem da técnica ${ }^{(9)}$.

Existem grandes vantagens na utilização de métodos digitais tanto para diagnóstico quanto para auxílio na realização de técnicas cirúrgicas. Com uma documentação digital em uma escala 1:1 é possível proporcionar uma melhor relação tecido mole/ tecido duro, justificando a escolha da confecção e utilização de um guia cirúrgico associado à técnica 
de Caldwell-Luc ${ }^{(5)}$. O uso da tomografia computadorizada e a confecção de protótipos de forma rápida proporcionam a precisão do planejamento e fidelidade da técnica, principalmente na instalação de implantes odontológicos. Na implantodontia a facilidade proporcionada é extremamente válida, pois permite cirurgias pouco invasivas, com frequência sem necessitar de retalhos, onde o guia é posicionado diretamente sobre a mucosa, favorecendo um melhor pósoperatório ${ }^{(16)}$.

O guia cirúrgico utilizado neste trabalho foi confeccionado a fim de proporcionar uma técnica minimamente invasiva e de alta previsibilidade, proporcionando ao paciente e à equipe cirúrgica, segurança durante o procedimento, diminuição do tempo operatório e, consequentemente, das complicações inerentes a esse tempo prolongado, e maior conforto pósoperatório. corpo estranho presente ou na prevenção de futuros processos inflamatórios crônicos ou agudos na cavidade.

O uso de planejamentos digitais e confecção de protótipos na odontologia proporcionam um momento cirúrgico mais eficiente, com um menor tempo cirúrgico, maior facilidade técnica e maior previsibilidade, proporcionando um pósoperatório mais confortável ao paciente e boa aceitação do paciente.

O guia idealizado de forma única e utilizado durante a técnica de Caldwell-Luc, para acessar o seio maxilar e realizar a remoção do corpo estranho, é vantajoso e pode ser reproduzível também para utilização em ambiente ambulatorial.

\section{Conclusão}

A remoção de terceiro molar no interior do seio maxilar se faz necessária, seja no tratamento de sinusites causadas pelo

\section{Referencias}

1. Oliveira RS, Costa RO, Neto LGC, Araújo FF. Aplicação da técnica cirúrgica de CaldwellLuc para remoção de corpo estranho do seio maxilar: relato de caso. J Health Sci Inst. 2010;28(4):318-20.

2. Batista PS, Júnior AFDR, Wichnieski C. Contribuição para o estudo do seio maxilar. Revista Portuguesa de Estomatología, Medicina Dentária e Cirurgia Maxilofacial. $2011 ; 52(4)$ 235-239.

3. Cerqueira LS, Almeida ADS, Rebouças DS, Sodré JS, Marchionni AMT. Remoção de corpo estranho em seio maxilar: relato de caso. Revista de Cirurgia e Traumatologia Bucomaxilo-facial. 2016; 16(2), 44-47.

4. Rebouças, D.S et al. Sinusite Crônica Decorrente de Corpo Estranho em Seio Maxilar. Relato de Caso. Revista Bahiana de Odontologia. 2014 Ago;5(2):131-136.

5. Camardella LT, Ongkosuwito EM, Waard O Breuning KH. A utilização do fluxo de trabalho digital no tratamento ortodôntico e ortocirúrgico. Orthod. Sci. Pract. 2015; 8(31):305 314.

6. Gagnier JJ, Riley D, Altman DG, Moher D, Sox $\mathrm{H}$, Kienle G; CARE Group. The CARE guidelines: consensus-based clinical case reporting guideline development. Dtsch Arztebl Int. 2013; 110 (37): 603-608.

7. Alves IFDS et al. Remoção de corpo estranho do seio maxilar por meio de corticotomia: Relato de caso clínico. 2015.

8. Hipolito II, Silva PFB, \& Gottardo VD. Complicação cirúrgica na instalação de implantes em proximidade com o seio maxilar: relato de caso. revista uningá. 2018; 55(2), 242-252.

9. Bellotti A, Costa FS, Camarini ET. Deslocamento de terceiro molar superior para o seio Maxilar: relato de caso. Rev. Cir. Traumatol. Buco-Maxilo-fac., Camaragibe 2008. v.8, n.4, p. $35-40$.

10. Meneses RO, Costa MCF, Lourenço RD, Cavalcante JR. Deslocamento de resto radicular no seio maxilar devido a manobras incorretas de exodontia: um relato de caso. Rev. Cir. Traumatol. Buco-Maxilo-Fac., Camaragibe 2014. v.14, n.4, p. 77-80.

11. Mariano RC, Melo WMD, \& Mariano LDCF. Introdução acidental de terceiro molar superior em seio maxilar. Revista de Odontologia da Universidade Cidade de São Paulo, 2006; $16(2), 167-70$

12. Gassen HT, Biancon Filho LA, Ciprandi MTO,
Silva-Júnior AN, \& Hernandez PAG. Deslocamento de corpo estranho para o seio maxilar: fatores etiológicos e remoção pela técnica de Caldwell-Luc. Revista Odontológica do Brasil Central, 2010; 16(42).

3. Graziani M. Cirurgia bucomaxilofacial. 8. ed. Rio de Janeiro: Guanabara Koogan, p. 401-3, 1995.

14. Martorelli SB, Andrade FBM, Cunha MB, Fonseca FLDMA. Sinusite Maxilar latrogênica por tratamento endodôntico: Revisão de literatura e relato de caso. Revista científica da OARF, 1(2). 2017.

15. Barbosa S, Polo TOB, Momesso GAC, Sousa CA, Silva WPP, Bassi APF, \& Faverani LP. Feixe de luz como auxiliar na remoção de corpo estranho no seio maxilar. Inovação técnica em cirurgia e implantologia. archives of health investigation, 2019; 7 .

16. Fonseca JM, Barbosa S, Momesso GAC, Polo TOB, Bassi APF, \& Faverani LP. Remoção de raiz dentária impelida no seio maxilar com o uso de fibra ótica. Relato de caso. archives of health investigation. 2017. 